\title{
RESEARCH
}

\section{PSYCHOMETRIC PROPERTIES OF THE TURKISH VERSION OF THE REVISED SCALE FOR CAREGIVING SELF-EFFICACY}

Turkish Journal of Geriatrics

DOI: 10.31086/tjgeri.2021.224

2021; 24(2): 276-286

\section{-Ecem ÖZGÜL ${ }^{1}$}

- Burcu AKPINAR SÖYLEMEZ2

CORRESPONDANCE

\section{${ }^{1}$ Ecem ÖZGÜL}

Izmir University of Economics, Nursing, İzmir, Turkey

\section{Phone: +902324888341}

e-mail: ozgulecem24@gmail.com

Received: Mar 24, 2021

Accepted: May 21, 2021

${ }^{1}$ Izmir University of Economics, Nursing, Izmir Turkey

${ }^{2}$ Dokuz Eylül University, Nursing, Izmir, Turkey

\section{Abstract}

Introduction: The self-efficacy of caregivers is an important matter that merits investigation, and this requires that the concept of self-efficacy be measured with a valid, reliable instrument.

Materials and Methods: This research examined the psychometric properties of the Turkish version of the Revised Scale for Caregiving SelfEfficacy. A sociodemographic form and the Revised Scale for Caregiving SelfEfficacy were employed to collect data from April through December 2019. A total of 250 family caregivers were included in the study, which assessed the content validity, construct validity and reliability of the Revised Scale for Caregiving Self-Efficacy.

Results: All 18 items had significant item-to-total correlations $(p<0.05)$. The Cronbach's alpha coefficient was 0.82 for the total scale, 0.76 for the selfefficacy in obtaining respite subscale, 0.82 for the self-efficacy in responding to disruptive patient behaviours subscale and 0.96 for the self-efficacy in controlling upsetting thoughts about caregiving subscale.

Conclusion: The results of this study indicate that the Revised Scale for Caregiving Self-Efficacy is a valid, reliable measurement tool and suitable to the Turkish culture.

Keywords: Dementia; Caregivers; Self Efficacy; Reproducibility of Results 


\section{INTRODUCTION}

The elderly population has grown significantly worldwide, accompanied by a rapid increase in the incidence of the chronic disease of dementia. According to the World Health Organization approximately 50 million people have dementia globally, and 10 million new cases are detected each year (1). A global prevalence study investigating countries and regions in the years 1990-2016 found that 754,169 individuals had dementia in Turkey, 35,355 had lost their lives to the disease and 462,429 had suffered from disabilities associated with dementia (2). There were no current data from Turkey in the Global Dementia Observatory country profiles (2017-2019) in 2020.

Dementia is a chronic, progressive syndrome that causes deterioration in cognitive function beyond what is expected from normal aging, affecting memory, thinking, orientation, comprehension, calculation, learning capacity, language and judgement (1). As dementia progresses, the care needs of people with dementia (PwD) also gradually increase. The care of the individual and the management of the disease are mostly provided by families in a home setting. The Alzheimer's Association (AA) reports that most caregivers (66\%) live with the person with dementia in the community (3).

It is well known that the care needs of dementia are complex, wide-ranging and extremely challenging (3-5). In caregiving, caregivers provide material, emotional, social and financial support to the person with dementia (6). Due to the challenges associated with caregiving, caregivers experience problems including the burden of care, emotional stress, depression, anxiety, negative family relationships, an increased risk of chronic diseases (such as hypertension and diabetes), sleep problems, smoking, dementia, job losses, financial difficulties and poor quality of life (6-9). The negative consequences of caregiving affect caregivers' own self-care behaviours and self-efficacy $(10,11)$.

The profiles of caregivers also affect their self- care and self-efficacy. AA reports that approximately two-thirds of dementia caregivers are women. About $30 \%$ are aged 65 or older, and over $60 \%$ are married, living with a partner or in a long-term relationship. Over half provide assistance to a parent or in-law with dementia. Among primary caregivers of PwD (i.e., those who indicate having the greatest responsibility for helping their relatives), over half care for parents. Approximately one-quarter of dementia caregivers are 'sandwich generation' caregivers, meaning that they care not only for an aging parent but also for a child (3). These profile characteristics complicate caregivers' daily activities, causing them to neglect their own care and experience difficulties in managing the disease. Studies in the literature have identified the factors affecting the self-efficacy of family caregivers $(4,5,12)$, confirming that their self-efficacy is a vital concern that merits evaluation to empower caregivers and reduce the negative consequences of care.

Self-efficacy is defined as people's desirable performance of their own activities and their management of situations encountered in daily life (13-15). The concept of self-efficacy, an element of Bandura's social cognitive theory, is frequently cited in studies (13-15). When caregivers face problems, their self-efficacy decreases, and negative thoughts increase. As this concept gains importance, studies of family caregivers' self-efficacy have proliferated $(16,17)$, and studies offering valid, reliable measurement tools to evaluate this concept are needed. The Revised Scale for Caregiving Self-Efficacy (RSCE), developed by Steffen et al. in 2002, is one scale used to examine the self-efficacy of caregivers of PwD (18). In 2018, Steffen et al. undertook a cross-national review and found that validity and reliability studies of this scale had been conducted in languages including Arabic, Chinese, English, French, Italian and Spanish) (17), but no psychometric analysis of the scale has been conducted in Turkish society. Accordingly, this research examined the psychometric properties of the Turkish version of the RSCE. 


\section{MATERIALS AND METHODS}

\section{Design and Sample}

A methodological, descriptive and correlational study design was used. The sample size suggested for studies of scale validity and reliability is 5 times the number of items in the scale (19), and in this case an estimated sample size of 90-180 represented between 5 and 10 times the 18 items in the scale. In April-December 2019, 250 family caregivers of PwD were recruited from a neurology and geriatrics outpatient clinic by non-probability convenience sampling. Random sampling was used to select the participants, who voluntarily agreed to take part. In the collection of research data, 250 caregivers met the inclusion criteria and were included in the analyses. The inclusion criteria were as follows: primarily responsible for a PwD; responsible for providing care for at least 1 year; providing $\geq 5$ hours of care per day; literate; can speak Turkish; willing to participate.

\section{Measures}

\section{Sociodemographic Form}

The form was developed by the researchers to collect data on the sociodemographic characteristics of caregivers and patients. The form composed of two parts. The first part contained questions regarding the caregivers' age, gender, educational status, relationship with the patient, working status, economic status, and the length of time of care. The second part contained questions about the PwDs' age, gender, education status, length of illness, the type and stage of dementia, and their capability of performing daily life activities.

\section{The Revised Scale for Caregiving Self-Efficacy}

The scale, originally called the Self-Efficacy Scale for Care, was developed in 1999 by Zeiss et al. to evaluate caregivers' self-efficacy and problem-solving self-efficacy (20). In 2002, the RSCE was revised by Steffen et al. to include 18 items measuring the self-efficacy of those caring for PwD. Validity and re- liability analyses were conducted (18). All the scale's questions aim to evaluate how effectively the caregiver achieves a particular goal. The 18 items are each scored from 0 through 100 points, with high scores indicating high self-efficacy. The scoring is interpreted as follows: $0 \%$ confidence indicates that the caregiver has low self-efficacy for an item, 50\% confidence indicates moderate self-efficacy and $100 \%$ confidence indicates high self-efficacy (18).

The 18-item scale contains three revised subscales: self-efficacy in obtaining respite (first subscale) (5 items), self-efficacy in responding to disruptive patient behaviours (second subscale) (8 items) and self-efficacy in controlling upsetting thoughts about caregiving (third subscale) (5 items). The Cronbach's alphas of the scale were 0.85 for the self-efficacy in obtaining respite subscale, 0.82 for the self-efficacy in responding to disruptive patient behaviour subscale and 0.85 for the self-efficacy in controlling upsetting thoughts about caregiving subscale (18). The current study tested the revised version of the RSCE. A researcher read the scale to the caregivers and recorded their answers. The validated scale questions in Turkish are provided in Appendix 1.

\section{Psychometric Analyses}

The Statistical Package for the Social Sciences version 21.0 (SPSS Inc, Chicago, IL, USA) and AMOS 24.0 were used for statistical evaluation of the data.

\section{Validity Analysis}

\section{Language and concept validity}

For language validity, the scale was independently translated from English to Turkish by two experts (an English language specialist and a subject matter expert whose native language is Turkish). Thereafter, the researchers collaborated to develop the Turkish version of the scale by determining the most appropriate translation for each item. The back translation of the scale from Turkish to English was done by a language expert who had 
not read the English version before and who had a good understanding of both languages and cultures. The translated English form and the original form were compared by the researchers. No items were changed.

\section{Content validity}

Content validity was confirmed by seven experts in dementia care (three psychiatric nurses, three internal medicine nurses and one psychologist), who were asked to evaluate the scale in terms of language and content. Their opinions were assessed using the Polit-Beck content validity index. The scale-level content validity index (S-CVI) and item-level content validity index (I-CVI) were calculated. The experts rated the items as 1 ('not appropriate'), 2 ('should be made more appropriate'), 3 ('appropriate but needs minor changes') or 4 ('highly appropriate'). The number of experts who score three or four is divided by the total number of experts to calculate the CVI. A CVI score above $80 \%$ indicates excellent agreement (21).

\section{Pilot study}

To test the clarity and intelligibility of the items, the scale was given to a small representative group of the sample. In this pilot study, a sample of 25 family caregivers was selected in light of the number of items and the intended sample size. The pretest sample data were not included in the study (19).

\section{Construct validity}

Confirmatory factor analysis (CFA) determined the construct validity. In the CFA, the authors analysed Pearson's $\chi^{2}$, degree of freedom, root mean square error of approximation (RMSEA), goodnessof-fit index (GFI), comparative fit index (CFI) and normal fit index as the GFIs.

\section{Reliability Analysis}

Reliability was determined using Cronbach's alpha, item-total correlations, test-retest, ceiling and floor effects and Hotelling's T-squared test for response bias (22).

\section{Ethical Consideration}

Written permission of RSCE was obtained via e-mail from Ann M. Steffen, who revised the scale in 2002, to perform the adaptation. Written permission was received from the Neurology and Geriatrics Department of $X$ University Hospital and Ethics Committee of $X$ University (approval number: 2019/01-04). Individuals were informed about the aim and design of the study, and their oral and written consents were obtained.

\section{RESULTS}

Family caregivers were mostly female (70\%), with age at the range of 26-78 years old with a mean of 54.29 years (standard deviation: 8.64). Of the caregivers, $54 \%$ were children of PwD.

\section{Validity}

\section{Content and language validity}

The opinions of seven experts were sought for language and concept validity. For all the items, the concordance of the experts' opinions was 0.94 for the whole scale.

\section{Confirmatory factor analysis}

The CFA identified the factor loadings of all the items in the scale. The factor loads in the first subscale ranged from 0.37 to 0.94 , those in the second subscale from 0.47 to 0.81 and those in the third subscale from 0.83 to 0.97 . The model fit indicators were determined as: $\mathrm{CFI}=0.93$, incremental fit in$\operatorname{dex}(\mathrm{IFI})=0.93, \mathrm{GFI}=0.92$, root mean square residual (RMR) $<0.01$, chi-square/degree of freedom $\left(x^{2}\right)$ $d f)=2.90(p<0.001)$ and $90 \%$ confidence interval of RMSEA $=0.07$ (Figure 1, Table 1).

\section{Reliability}

\section{Test-retest reliability}

Before the data collection, the scale was provided to 25 randomly selected family caregivers, and a 2- to 6-week period passed between the two 
Figure 1. Confirmatory Factor Analysis Results of the Revised Scale for Caregiving Self Efficacy
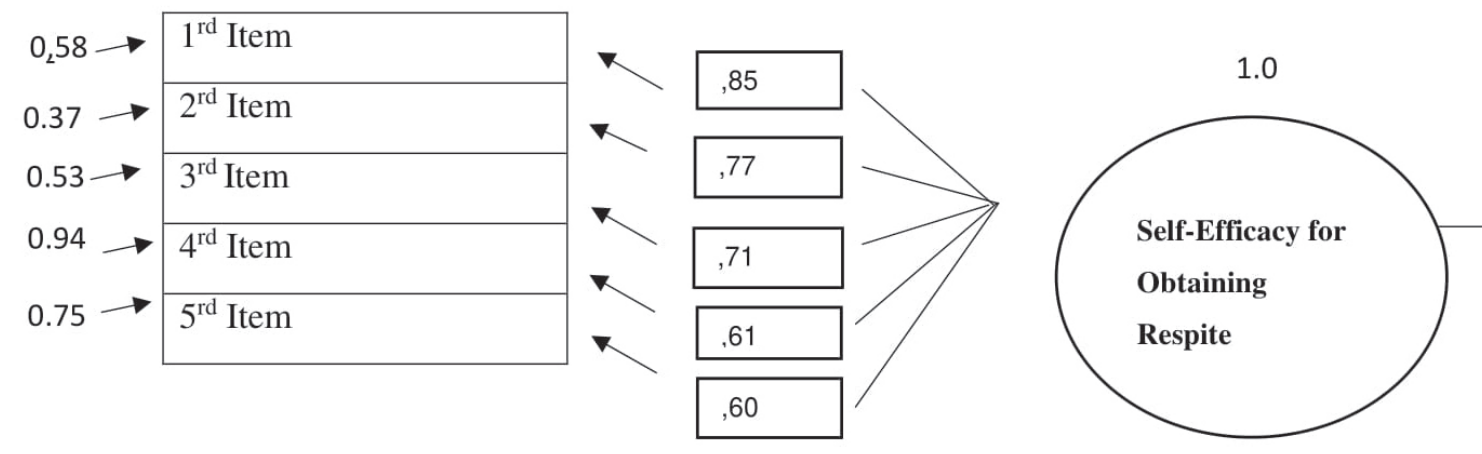

$-, 11$

\begin{tabular}{|c|c|}
\hline $0.58 \rightarrow$ & $6^{\text {rd }}$ Item \\
\hline $0.51 \longrightarrow$ & $7^{\text {rd }}$ Item \\
\hline $0.47 \rightarrow$ & $8^{\text {rd }}$ Item \\
\hline $0.56 \rightarrow$ & $9^{\text {rd }}$ Item \\
\hline $0.67 \rightarrow$ & $10^{\text {rd }}$ Item \\
\hline $0.66 \longrightarrow$ & $11^{\text {rd }}$ Item \\
\hline $0.81 \rightarrow$ & $12^{\text {rd }}$ Item \\
\hline $0.74 \longrightarrow$ & $13^{\text {rd }}$ Item \\
\hline
\end{tabular}
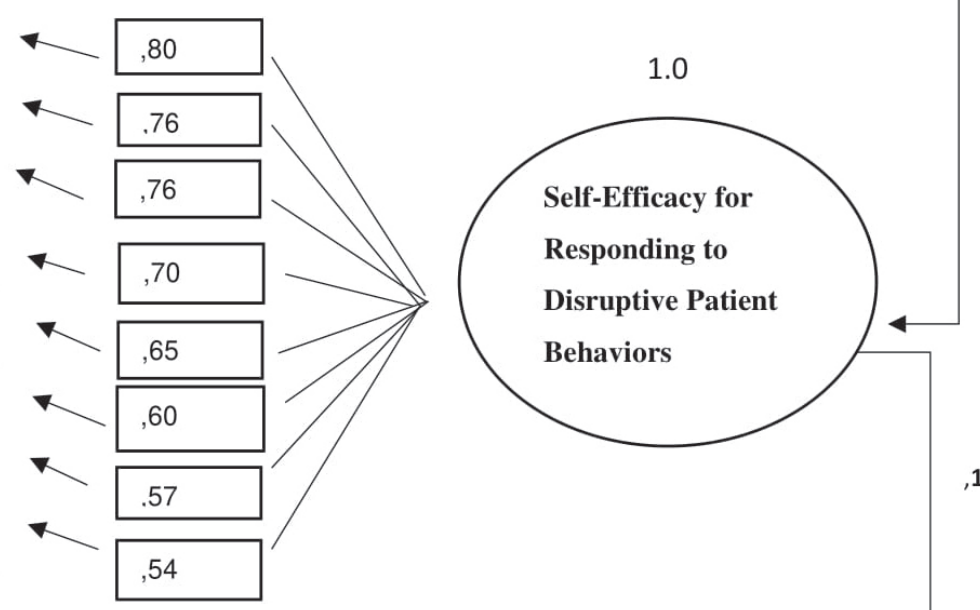

, 13

,16

1.0
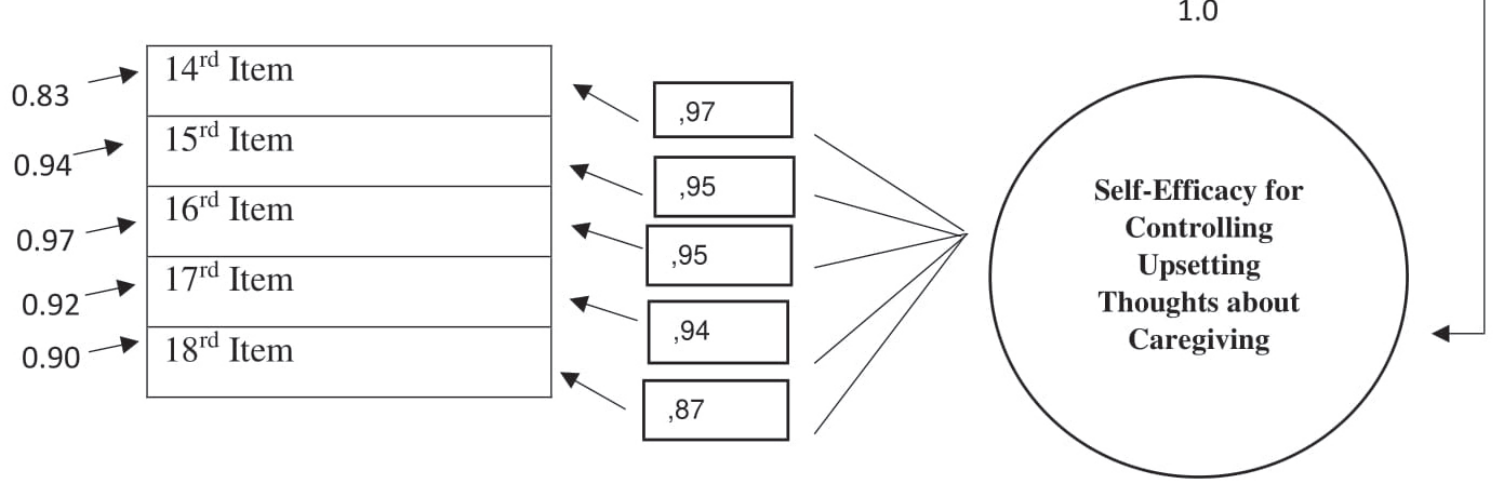

Chi-Square: 2.90 p-value: 0.01, RMSEA: 0.07 GFI:0.92, CFI:0.93, IFI:0.93 
Table 1. Model fit indices of confirmatory factor analysis of the RSCE

\begin{tabular}{|l|c|c|}
\hline \multicolumn{1}{|c|}{ Model fit indices of CFA } & Reference values ${ }^{\text { }}$ & Values in the current study \\
\hline$\chi^{2} /$ df (Chi-Square/Degree of Freedom) & $<5$ & 2.90 \\
\hline GFI (Goodness of Fit Index) & $>0.90$ & 0.92 \\
\hline IFI (Incremental Fit Index) & $>0.90$ & 0.93 \\
\hline CFI (Comparative Fit Index) & $>0.90$ & 0.93 \\
\hline RMSEA (Root Mean Square Error of Approximation) & $<0.08$ & 0.07 \\
\hline RMR (Root Mean Square Residual) & $<0.08$ & $<0.01$ \\
\hline
\end{tabular}

a Peñacoba C, Losada A, López J, Márquez-González M. Confirmatory factor analysis of the Revised Scale for Caregiving Self-Efficacy in a sample of dementia caregivers. International Psychogeriatrics 2008;20: 1291-1293. (PMID: 18620625).

data collections. A statistically significant positive relationship was observed between the test-retest scores of the scale's three subscales. The first subscale had an $r=0.99$ and a $p<0.001$, the second had an $r=0.96$ and a $p<0.001$ and the third had an $r=0.98$ and $a p<0.001$.

\section{Internal consistency analysis}

The total Cronbach's alpha internal consistency reliability coefficient was 0.82 , and the Cronbach's alpha coefficient was 0.76 for the first subscale, 0.82 for the second and 0.96 for the third. The item-total correlations were statistically significant $(p<0.05)$ and varied from 0.46 to 0.68 for the first subscale, 0.42 to 0.67 for the second and 0.81 to 0.95 for the third.

\section{Floor and ceiling effect analysis}

When the data were evaluated, the floor and ceiling effect of the scale was less than 15\%. Response bias was evaluated to test whether the participants answered in line with their own opinions or with the expectations of the community or researcher while completing the scale. The Hotelling T2 value was 29.823 and was significant $(p<0.001)$.

\section{DISCUSSION}

\section{Validity}

In the current study, a Turkish version of the RSCE was prepared, and the language validity criterion was fulfilled (the first step in scale adaptation studies). The CFA yielded factor loads from 0.37 to 0.94 in the first subscale and $0.47-0.81$ and $0.83-0.97$ in the second and third subscales, respectively. The CFA determined that the factor loads of all the subscales were above 0.30 . The Turkish version of the scale is structurally similar to the original, so the structural validity of the Turkish version was confirmed. The results of the CFA in this study support the construct validity of the scale, confirming its validity. The present study also supports two CFA studies $(23,24)$ that confirmed the three-factor structure identified in the original development article.

An RMSEA value of 0.08 or less is considered an adequate fit, with RMSEA $=0$ indicating a perfect fit. When the model fit indicators were examined in the current study, the RMSEA value of the scale was found to be 0.07 , indicating that the model ad- 
equately agrees with the sample. When the GFI is $>0.90$, the factor model well explains the original variability, and the model is usable, with GFI values close to 1 indicating a good fit. In the current study, the GFI value was above 0.92 , indicating that our scale has good fit-index values. IFI and CFI values of $>0.90$ indicate an acceptable fit, and those values in this study were found to be 0.93 .

A high correlation between the observed variables causes the chi-square value to increase. The ratio of the degrees of freedom to the chi-square, an important criterion of the chi-square test, can be used as a fit criterion. A ratio of less than 5 is considered to indicate a good fit (25). In this study, the $\mathrm{x}^{2} / \mathrm{df}$ ratio was 2.90 , and the RMR value, an absolute index, was 0.01 , indicating strong fitness. The RSCE confirmed its construct validity by providing five fit indexes, and these results agree with those of other language adaptations $(23,24)$. The CFA results of the Chinese version of the scale were $x^{2}=29.09$, df $=23$ and RMSEA = 0.05 (23). The CFA of the Spanish version of the scale supported the three-factor model $\left(x^{2}=120.86, \mathrm{df}=87, \mathrm{GFI}=0.93, \mathrm{IFI}=\right.$ $0.97, \mathrm{CFI}=0.97, \mathrm{RMSEA}=0.04$; the factor loadings ranged from 0.45 to 0.94 ) (24).

\section{Reliability}

The test-retest reliability analysis of the RSCE was evaluated by Pearson correlation analysis, which yielded a correlation coefficient of 0.70-1.00. This value is sufficient to confirm a high reliability. The test-retest reliability analysis results of this study are compatible with those of the original scale, which were above 0.70 for all the subscales (18).

A statistically significant positive relationship was observed between the test-retest scores of the scale's three subscales $(r=0.99$ and $p<0.001$ for first subscale, $r=0.96$ and $p<0.001$ for the second and $r$ $=0.98$ and $p<0.001$ for third). A T-test was conducted on the dependent groups to determine whether a difference exists between the mean scores obtained after repeated measurements of the scale. No statistically significant difference was observed between the mean scores $(p>0.05)$.
To confirm a scale's reliability, all its subscales should be used to measure the same property. Therefore, the reliability coefficient of the scale should be calculated. Cronbach's alpha values between 0.60 and 0.80 indicate that a scale is fairly reliable, and values between 0.80 and 1.00 indicate that a scale is highly reliable (22). The first subscale's Cronbach's alpha was $a=0.76$, the second's $a=0.82$ and the third's $a=0.96$; the whole-scale alpha was a $=0.82$. These results indicate that the full scale and its subscales are highly reliable and have a strong internal consistency. Furthermore, in the original study, the Cronbach's alphas were above 0.80 (18). Our results agree with those of the English as well as those of the Chinese version (in which the Cronbach's alphas of the first, second and third subscales were $0.92,0.86$ and 0.75 , respectively) (23).

Regarding item reliability, an item-total analysis was conducted to confirm that the items in the subscales measure the concepts they are intended to measure. An item-total analysis score should be greater than 0.25 for each item (22). The item-total analysis yielded item-total scores above 0.40 for all the subscales, indicating that all the scale items had a high correlation with the total score and with the total scores of the subscales. The item reliability of the Turkish version of the scale, with factor loadings of 0.42-0.95, agrees with those of the English (factor loadings of $0.30-0.94$ ) and Spanish (factor loadings of 0.45-0.94) versions of the scale (24).

On the basis of the total score, the lowest total score obtainable from a scale indicates the floor effect, and the highest total score indicates the ceiling effect. The floor and ceiling effects should not exceed $15 \%$ (22). The analysis demonstrated the homogeneity of the scale. Hotelling's T-squared test was used to determine response bias. This test also indicates whether the average of the whole is normally distributed. The test's score is expected to be statistically significant to avoid response bias ( $p$ $<0.05)(22)$. In this test, the Hotelling's $T^{2}$ of the RSCE was 29.823 and determined as $p<0.001$, confirming 
the absence of reaction bias in the scale. The original study was evaluated as lacking information on the floor and ceiling effects and on responsiveness measurement domains.

\section{CONCLUSION}

The results of this study confirm that the RSCE is a valid, reliable measurement tool to assess the self-efficacy of family caregivers of PwD in the Turk-

\section{REFERENCES}

1. World Health Organization. Fact sheets; 2020. [Internet]. Available from: https://www.who.int/ news-room/fact-sheets/detail/dementia Accessed:22.01.2021.

2. Nichols E, Szoeke CE, Vollset SE et al. Global, regional, and national burden of Alzheimer's disease and other dementias, 1990-2016: a systematic analysis for the Global Burden of Disease Study 2016. The Lancet Neurology 2019;18(1):88-106. (PMID: 30497964)

3. Alzheimer's Association. Alzheimer's disease facts and figures [e-book]; 2020. [Internet]. Available from: https://www.alz.org/media/Documents/alzheimersfacts-and-figures.pdf. Accessed: 17.02.2021.

4. Tan GTH, Yuan Q, Devi F et al. Factors associated with caregiving self-efficacy among primary informal caregivers of persons with dementia in Singapore. BMC Geriatrics 2021;21(1):13. (PMID: 33407201)

5. Tonga JB, Eilertsen DE, Solem IKL, Arnevik EA, Korsnes MS, Ulstein ID. Effect of self-efficacy on quality of life in people with mild cognitive impairment and mild dementia: the mediating roles of depression and anxiety. American Journal of Alzheimer's Disease \& Other Dementias 2020;35:1533317519885264. (PMID: 31916847)

6. Lindeza P, Rodrigues M, Costa J, Guerreiro M, Rosa MM. Impact of dementia on informal care: a systematic review of family caregivers' perceptions. BMJ Supportive \& Palliative Care 2020;0:1-12. (PMID: 33055092)

7. Igarashi A, Fukuda A, Teng L, Ma F, Dorey J, Yoshie O. Family caregiving in dementia and its impact on ish culture. Given the importance of family caregiver interventions in the global community, the use of this scale should benefit family caregivers.

\section{Acknowledgements}

The authors thank the caregivers of PwD who participated in this study.

\section{Declaration of Interest Statement}

No conflict of interest has been declared by the authors.

quality of life and economic burden in Japan-web based survey. Journal of Market Access \& Health Policy 2020;8:1720068. (PMID: 32128076)

8. Laks J, Goren A, Dueñas H, Novick D, Kahle-Wrobleski K. Caregiving for patients with Alzheimer's disease or dementia and its association with psychiatric and clinical comorbidities and other health outcomes in Brazil. International Journal of Geriatric Psychiatry 2016;31(2):176-185. (PMID: 26011093)

9. Queluz F, Kervin E, Wozney L, Fancey P, McGrath $P$, Keefe J. Understanding the needs of caregivers of persons with dementia: A scoping review. International Psychogeriatrics 2020;32(1):35-52. (PMID: 30967164)

10. George NR, Steffen A. Physical and mental health correlates of self-efficacy in dementia family caregivers. Journal of Women \& Aging 2014;26:319-331. (PMID: 25133944)

11. MacDougall M, Steffen A. Self-efficacy for controlling upsetting thoughts and emotional eating in family caregivers. Aging \& Mental Health 2017;21 (10):1058-1064. (PMID: 27323869)

12. Cheng ST, Mak EP, Fung HH, Kwok T, Lee, DT, Lam LC. Benefit finding and effect on caregiver depression: A double-blinded randomized controlled trial. Journal of Consulting and Clinical Psychology 2017;85(5):521-529. (PMID: 28287803)

13. Bandura A. Social cognitive theory in cultural context. Applied Psychology: An International Review 2002;51:269-290. (DOI:10.1111/1464-0597.00092).

14. Bandura A. Self-efficacy beliefs of adolescents. In Pajares F. \& Urdan T. (Eds.). Guide to constructing self-efficacy scales. Charlotte: Information Publishing, 2006, pp 307-337. 
15. Bandura A. On the functional properties of perceived self-efficacy revisited. Journal of Management 2012;3:9-44. (DOI:10.1177/0149206311410606).

16. Crellin NE, Orrell M, McDermott O, Charlesworth G. Self-efficacy and health-related quality of life in family carers of people with dementia: A systematic review. Aging \& Mental Health 2014;18:954-969. (PMID: 24943873)

17. Steffen AM, Gallagher-Thompson D, Arenella K et al. Validating the revised scale for caregiving self-efficacy: A cross-national review. The Gerontologist 2019;59(4):325-342. (PMID: 29546334)

18. Steffen AM, McKibbin C, Zeiss AM, Gallagher-Thompson D, Bandura $A$. The revised scale for caregiving self-efficacy: Reliability and validity studies. Journal of Gerontology: Psychological Sciences 2002;57:74-86. (PMID: 11773226)

19. Sousa VD, Rojjanasrirat W. Translation, adaptation and validation of instruments or scales for use in cross-cultural health care research: a clear and user-friendly guideline. Journal of Evaluation in Clinical Practice 2011;17(2):268-74. (PMID: 20874835)

20. Zeiss AM, Gallagher-Thompson D, Lovett S, Rose J, McKibbin C. Self-efficacy as a mediator of caregiver coping: Development and testing of an assess- ment model. Journal of Clinical Geropsychology 1999;5:221-230. (DOI: 10.1023/A:1022955817074).

21. Polit DF, Beck CT, Owen SV. Is the CVI an acceptable indicator of content validity? Appraisal and recommendations. Res Nurs Health 2007;30(4):459-67. (PMID: 17654487)

22. Boateng GO, Neilands TB, Frongillo EA, Melgar-Quiñonez HR, Young SL. Best practices for developing and validating scales for health, social, and behavioral research: a primer. Frontiers in Public Health 2018;6:149. (PMID: 29942800)

23. Cheng ST, Lam LW, Kwok T, Ng NS, Fung AT. Self-efficacy is associated with less burden and more gains from behavioral problems of Alzheimer's disease in Hong Kong Chinese caregivers. The Gerontologist 2013;53(1):71-80. (PMID: 22563001)

24. Peñacoba C, Losada A, López J, Márquez-González M. Confirmatory factor analysis of the Revised Scale for Caregiving Self-Efficacy in a sample of dementia caregivers. International Psychogeriatrics 2008;20:1291-1293. (PMID: 18620625).

25. Hooper D, Coughlan J, Mullen MR. Structural equation modelling: Guidelines for determining model fit. The Electronic Journal of Business Research Methods 2008; 6 (1):53-60. (DOI: 10.21427/D7CF7R). 